\title{
SIL-2R- an Immuno-biomarker for Prediction of Metastases in Uveal Melanoma
}

\author{
VIVIAN BARAK ${ }^{1}$, INA KALICKMAN ${ }^{1}$ and JACOB PE'ER ${ }^{2}$ \\ ${ }^{1}$ Immunology Laboratory for Tumor Diagnosis and ${ }^{2}$ Ophthalmology Department, \\ Hadassah - Hebrew University Medical Center, Jerusalem, Israel
}

\begin{abstract}
Background/Aim: Uveal melanoma (UM) is the most common primary intraocular malignancy in adults. High serum levels of soluble $I L-2$ receptor ( $I L-2 R)$ have been reported in acute inflammations and metastatic cancers. This study evaluated the potential of high/increasing sIL-2R levels in predicting metastases. Patients and Methods: The study included a total of 1,546 sera samples of subjects from three groups: 119 healthy controls (73 subjects), 566 UM 10 year (10y) disease-free (DF) (220 patients), 861 metastatic UM (268 patients). Patients were followed-up biannually with liver ultrasound and liver function tests for the presence of metastases (Mets). Blood samples to measure the levels of sIL-2R were obtained at the time of primary diagnosis, soon after initial treatment (enucleation, brachytherapy), every 6 months, 10 years from diagnosis, at Mets confirmation by $C T$, and after additional treatments. Results: Significantly higher sIL-2R levels were detected in the Mets patients compared to healthy controls and 10y DF patients. Compared to the upper limit of the normal levels of $s I L-2 R, 1,000 \mathrm{U} / \mathrm{ml}$, its levels in metastatic UM were $61 \%$, 25\% in $10 y$ DF UM, and $6.25 \%$ in the controls. High levels of $s I L-2 R$ in metastatic patients, decreased significantly post treatments. Individual kinetics of markers, indicated similar trends of SIL-2R compared to osteopontin and S-Protein 100, predicting metastases, which were confirmed on liver imaging. Conclusion: Significantly higher sIL-2R levels were evident in all UM patients with
\end{abstract}

Correspondence to: Prof. Vivian Barak, Head, Immunology Laboratory for Tumor Diagnosis, Department of Oncology, Hadassah-Hebrew University Medical Center, Ein Kerem, P.O.B. 12000, Jerusalem 9112001, Israel. Tel: +972 0522401916, e-mail: barakvivi@hadassah.org.il,barakvivi@gmail.com

Key Words: sIL-2R, biomarker, metastases prediction, uveal melanoma.

This article is an open access article distributed under the terms an conditions of the Creative Commons Attribution (CC BY-NC-ND) 4.0 international license (https://creativecommons.org/licenses/by-nc-nd/4.0).
Mets. Significant increases in sIL-2R levels on serial evaluations indicated and predicted UM Mets, enabling earlier treatment of Mets, to improve survival.

Uveal melanoma (UM) is the most common primary intraocular malignancy in adults with an incidence of 6-8 new cases per million people, every year. The mortality of UM due to metastases (Mets), is about 20\% 5 years after diagnosis, reaching up to $50 \%$ in 15 years. The most common metastatic site of UM is the liver; in many cases it is the only affected organ. Accordingly, most ocular oncologists screen with liver function tests, and liver imaging [magnetic resonance imaging (MRI), computed tomography (CT) and ultrasound (US)], every 6-12 months. Unfortunately, metastases are discovered at a relatively late stage with already advanced liver disease $(1,2)$.

Therefore, there is a need for reliable, non-invasive and lowcost tumor markers with high sensitivity, for earlier detection of Mets in UM. We have previously identified potential effective markers for UM Mets detection (3-6) such as S-Protein 100 (S100), melanoma inhibitory activity (MIA), osteopontin (OPN), tissue polypeptide specific antigen (TPS), Vascular endothelial growth factor (VEGF), and insulin-like growth factor 1 (IGF1). A relatively new tumor marker for UM, not published earlier, is soluble IL-2 receptor (sIL-2R). The IL-2 receptor consists of 3 subunits, and the $\alpha$ subunit, meaning CD25, can be cleaved and released into the blood (7).

We and others have previously reported on high levels of sIL-2R in various lymphomas and leukemia (8-14), especially in patients with higher grade disease, with good correlation to treatment responses. sIL-2R has been reported to be an extremely strong prognostic factor for patients with peripheral T-cell lymphoma unspecified (13). In hairy cell leukemia, sIL$2 \mathrm{R}$ has been demonstrated to be an important biomarker for its detection, disease activity, response to treatment, and prediction of early progression of disease (10).

High levels of sIL-2R have been reported in various inflammation-related conditions, especially in advanced disease stage and acute-phase of diseases such as sepsis (15), throat infections (16), ocular Behcet's disease (17), and primary biliary cirrhosis (18). 

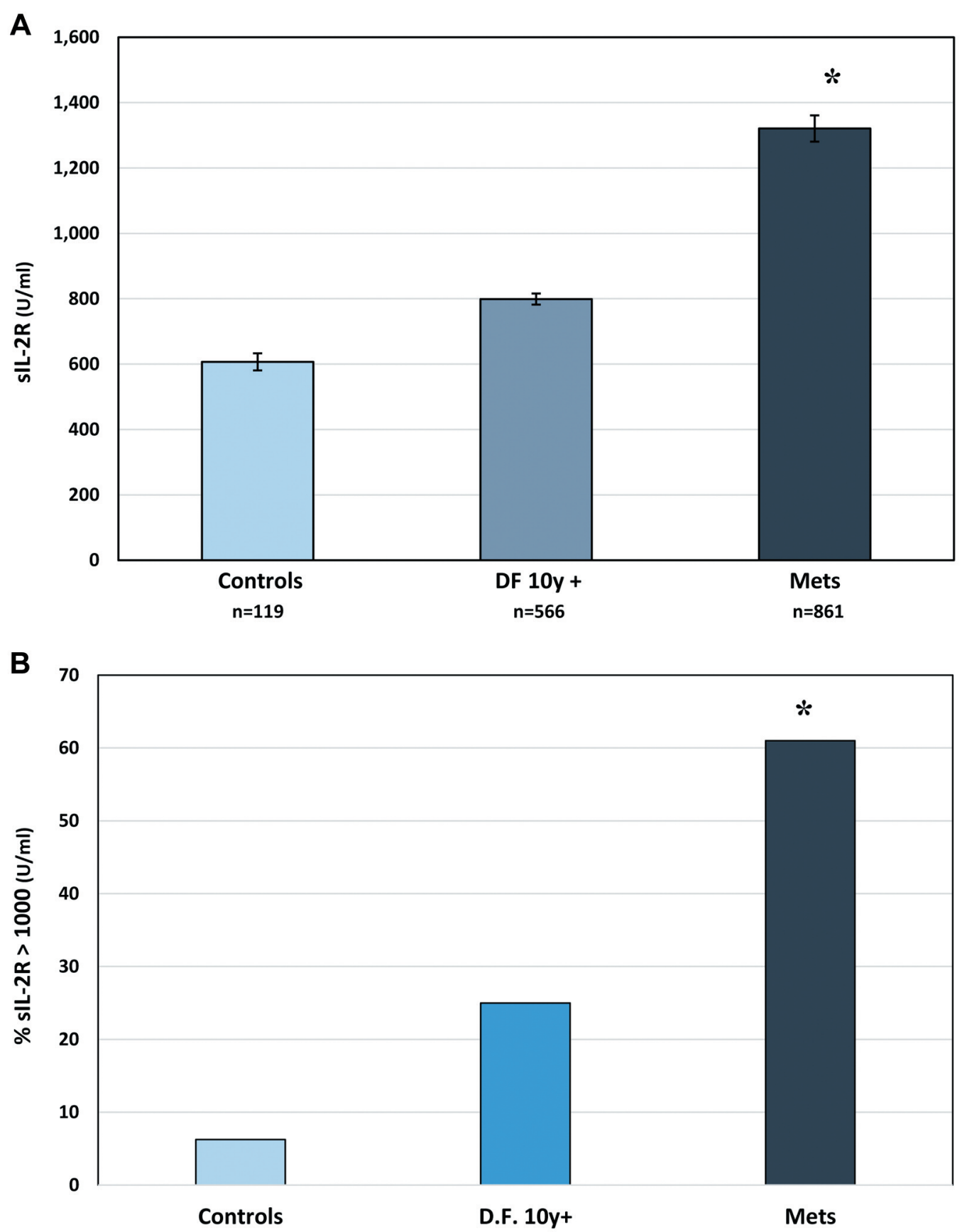

Figure 1. Levels of sIL-2R (A) and percentage of sIL-2R $>1,000(B)$ in uveal melanoma patients and controls. Significantly higher levels of sIL-2R and percentage of sIL-2R $>1,000 \mathrm{U} / \mathrm{ml}$ in metastatic and DF patients are shown, as compared to controls.

In several types of cancer, especially in the metastatic stage, high levels of sIL-2R have been reported, whereas a significant decrease has been observed after response to therapies. Furthermore, high levels of sIL-2R have been recorded in cutaneous melanoma (19), gastric cancer (20), and head and neck cancers $(21,22)$ compared to healthy controls.
We and others have also demonstrated that increasing levels of sIL-2R predicted metastases development and associated with a shorter survival time of patients. sIL2R has also been suggested as an independent prognosticator of Mets in many types of inflammatory diseases and cancer (19-22). 


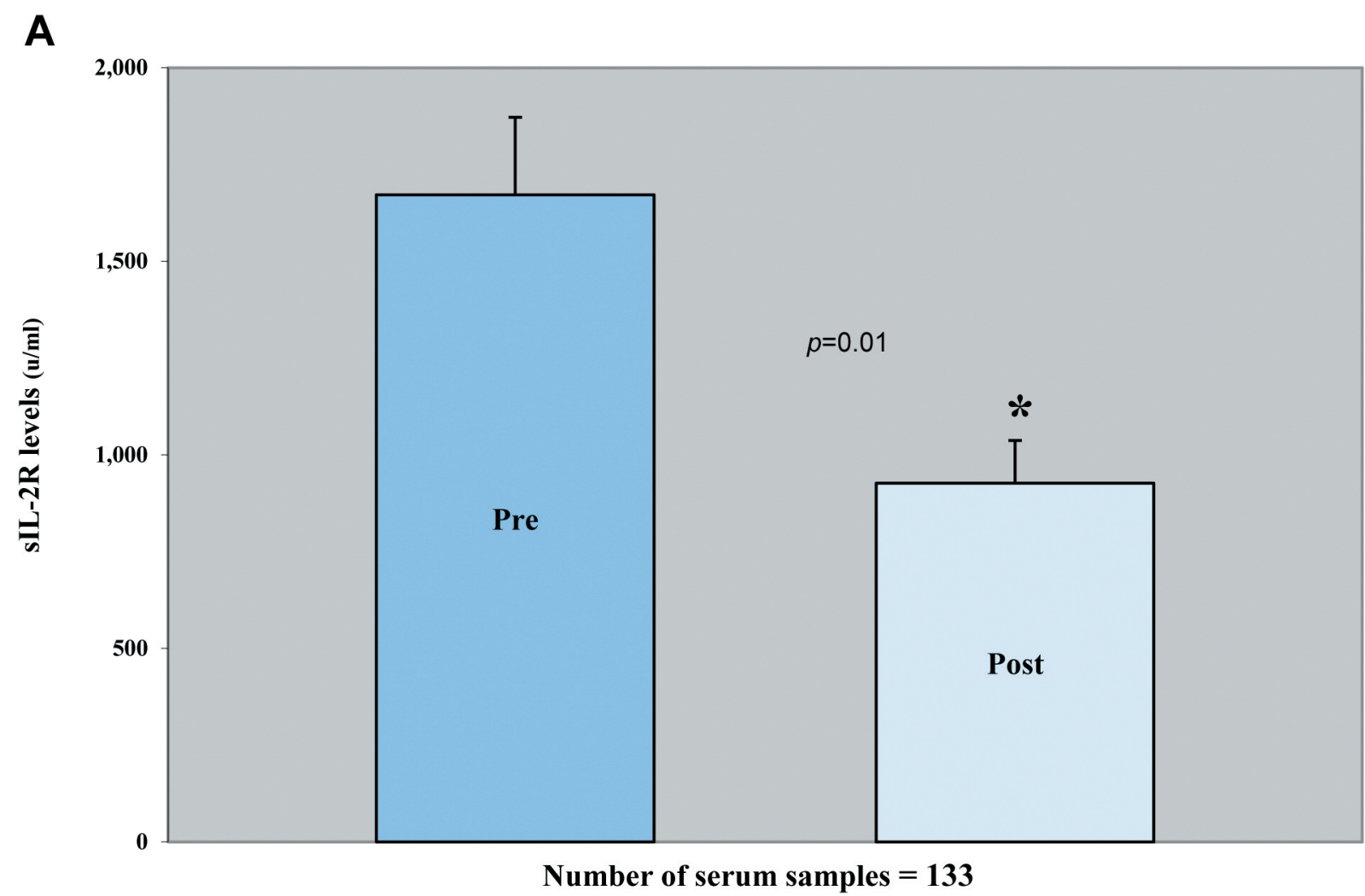

B
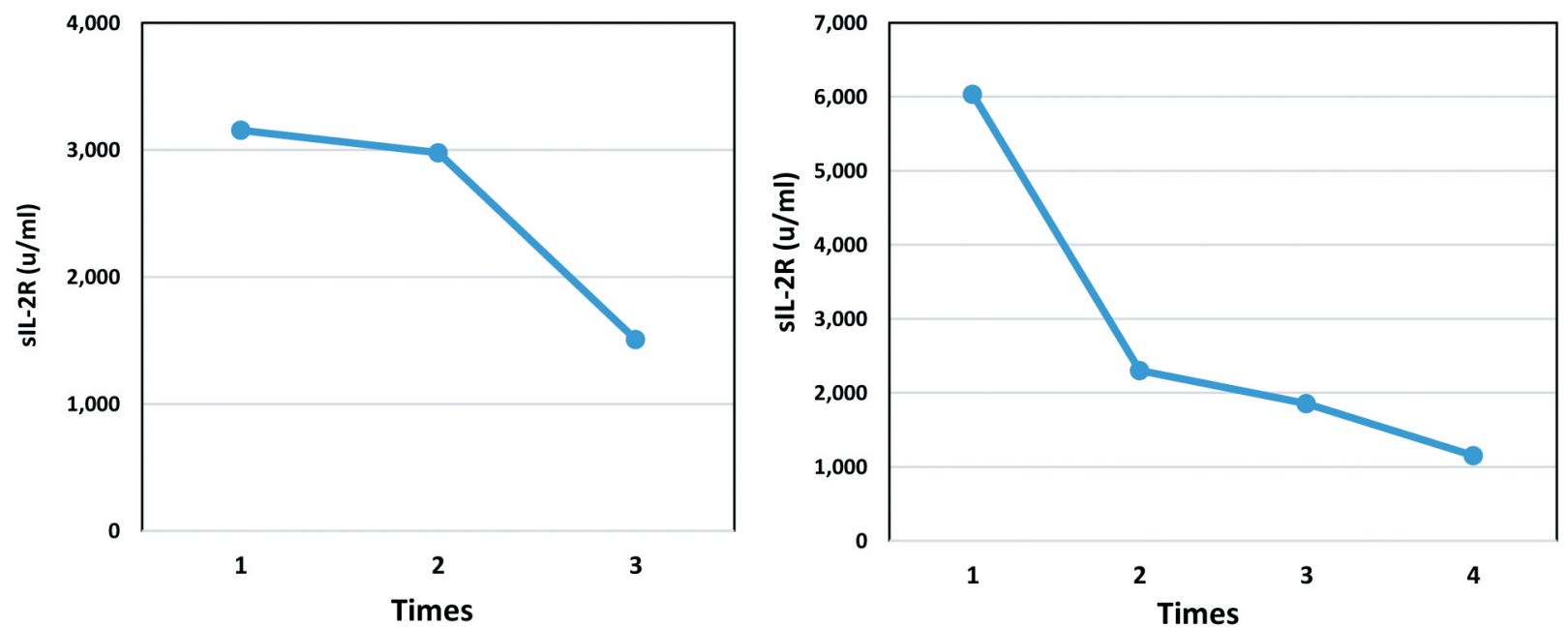

Figure 2. sIL-2R levels are significantly reduced post-treatment, as compared to those pre-treatment. (A) Effect of treatment (brachytherapy, stereotactic radiosurgery or enucleation) on sIL-2R levels in uveal melanoma patients, pre-and post-therapy. (B) Time-dependent decreases (every 2 months) in the levels of sIL-2R following treatment of individual uveal melanoma patients.

Major depressive disorder (MDD) and schizophrenia have been described to be associated with inflammatory processes. Serum levels of IL-6, IL-18, TNF $\alpha$, and sIL-2R were found to be elevated in depressed and schizophrenic patients, as compared to healthy controls. A significant increase $(p<0.05)$ in serum levels of cytokines in patients with MDD and schizophrenia, in spite of lacking an overt inflammation, have been reported. Therefore, anti-inflammatory drugs as adjuvant therapy in patients with schizophrenia and MDD, have been implemented (23-27). 


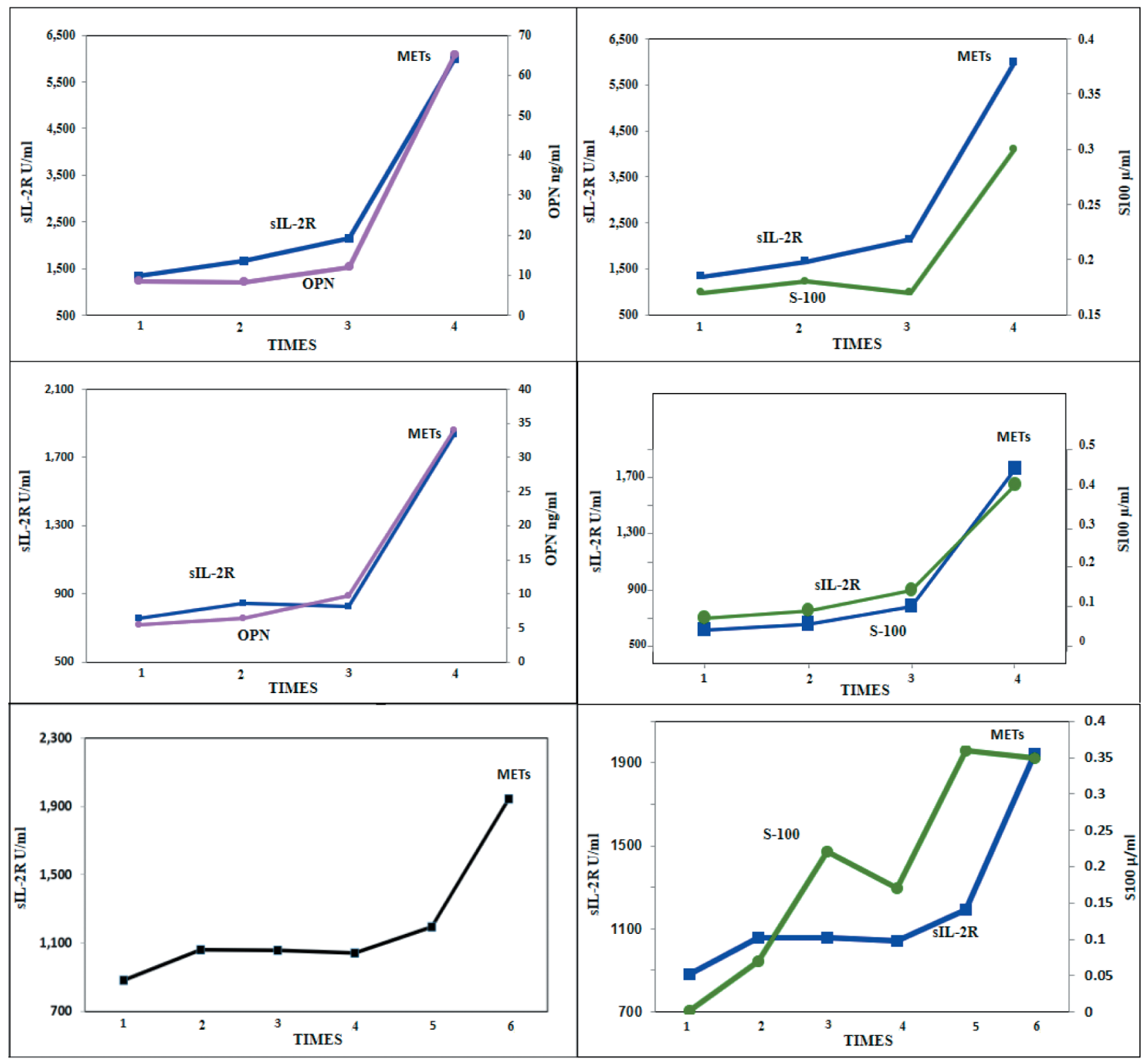

Figure 3. Metastases detection by sIL-2R increasing levels, in comparison to additional biomarkers: OPN and S-100, every 6 months of follow-up.

We have shown preliminary results on the role of sIL- $2 \mathrm{R}$ in UM patients (28). This study utilized a larger study population and compared other markers that we have previously shown to be effective biomarkers for predicting metastases in UM. The aim of this study was to evaluate the potential of high or increasing sIL-2R levels to predict UM Mets development.

\section{Patients and Methods}

Blood samples were obtained at the time of primary diagnosis, soon after initial treatment (enucleation, brachytherapy), in follow-up examinations every 6 months, at Mets confirmation, and after additional treatments for the ocular tumors. The study included a total of 1,546 serum samples of subjects from the 3 groups: 861 serum samples from Mets UM patients, 566 samples from UM patients 10y disease-free (DF) and 119 samples from healthy controls.

We included 73 healthy individuals, $22010 \mathrm{y}$ DF UM patients and 268 metastatic UM patients. Patients were followed up biannually with liver imaging and liver function tests for the presence of Mets. Serum levels of sIL-2R were analyzed by ELISA tests (Abbott GmbH., Wiesbaden, Germany).

Statistical analysis was performed by using the SPSS: IBM SPSS Statistics for windows, Version 25.0, 2017. IBM Corp., Armonk, NY, USA). 


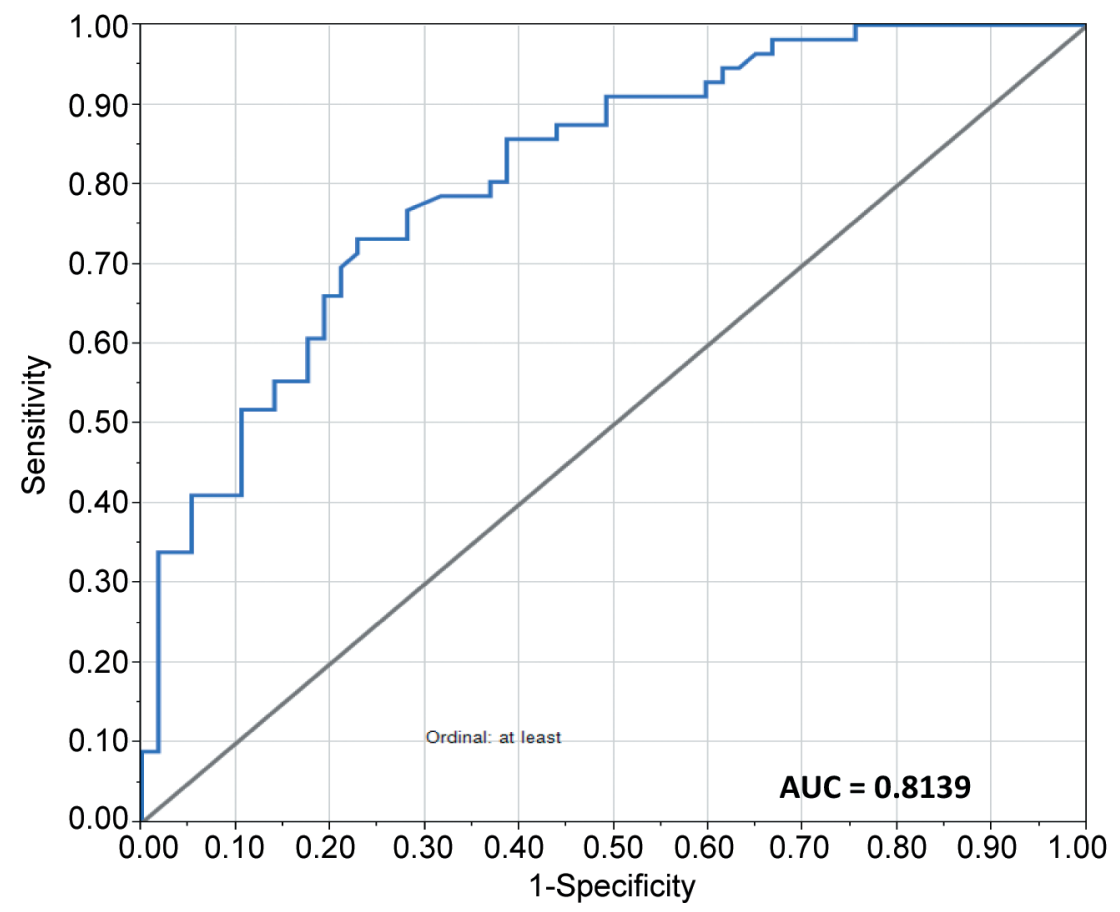

Figure 4. Receiver operating characteristic (ROC) analysis of sIL-2R levels for metastases prediction (AUC=0.8139).

\section{Results}

Significantly higher sIL-2R levels were detected in the Mets patients, compared to healthy controls and $10 \mathrm{y}$ DF patients, as shown in Figure 1A. The study included a total of 1,546 sera samples from subjects from 3 groups: 119 samples from 73 healthy controls, 566 samples from $22010 \mathrm{y}$ DF UM patients, and 861 samples from 268 metastatic UM patients. Percentage of sIL-2R sera levels $>1,000 \mathrm{U} / \mathrm{ml}$ (the cut-off between normal and pathological results) in each group, were as follows: metastatic patients showed $61 \%$ higher levels, 10y DF patients- 25\%, and controls - 6.25\% (Figure 1B). The increasing levels of sIL-2R predicted metastases formation, which was confirmed later by liver ultrasound, CT, or MRI.

The high levels of sIL-2R in metastatic UM patients decreased significantly following treatment of the ocular tumor (brachytherapy, stereotactic radiosurgery or enucleation) every 2 months, as shown in Figure 2.

The association of sIL-2R levels with disease and response to therapy was comparable to that of OPN and S100 , which were shown formerly to be sensitive markers in UM (Figure 3A). Significant increases in the levels of sIL$2 \mathrm{R}$, were very similar to those of OPN and S-100 and predicted metastases formation, as confirmed by liver ultrasound, CT and MRI, only a few months later (Figure 3B and C). Receiver operating characteristic (ROC) analysis is related in a direct and natural way to cost/benefit analysis of diagnostic decision making. ROC analysis since 1944, has been used in medicine, radiology, and biometrics. We performed ROC analysis of sIL-2R and showed (area under the curve $=0.8139$ ), which is presented in Figure 4.

\section{Discussion}

In this study, we have clearly demonstrated that there is a strong correlation between sIL-2R levels and the stage of UM disease: low levels were found in the Controls group and in the $10 \mathrm{y}$ DF patients. In contrast, in the metastatic group, almost all patients had high levels of sIL-2R. Increasing levels, as shown by the kinetics of changes in the levels of the marker were associated with and predicted metastases formation. These results are very similar to those found in other diseases, as we and others have shown in inflammatory conditions, several other metastatic cancers, and in MDD (8, $10,11,18,21,27)$. In our former studies on UM markers, S100 had a higher sensitivity in predicting disease than MIA and OPN by ROC analysis (3-5). In our preliminary study, as well as in this larger study, we have shown by ROC analysis that sIL-2R has a sensitivity of $81 \%$ (28). The effect of recent treatments of metastatic UM including chemotherapy, immunotherapy and new biological drugs, is questionable. Resection of isolated hepatic metastases can significantly prolong survival. However, in some cases, hepatic metastases occur in patients with normal serum enzymes (29). 
Therefore, the use of blood tumor markers, which are noninvasive and highly sensitive, is a superior way to detect early metastases, as we have shown previously with the markers S-100, MIA, OPN, and TPS $(3,4)$. However, we have also reported on markers that are not suggested to be used in routine clinical evaluations, such as VEGF and IGF1 , due to the great variability in their levels $(6,30)$.

\section{Conclusion}

Significantly higher serum sIL-2R levels were found in UM patients with metastases as compared to controls. In individual serial evaluations, significant increases in sIL-2R levels, may indicate the early development of UM metastases in such patients.

\section{Conflicts of Interest}

The Authors declare no competing interests regarding this study.

\section{Authors' Contributions}

BV: Project PI and paper preparation. KI: Technical assistance. PJ: Clinical PI.

\section{Acknowledgements}

The Authors thank Shani Cohen for the technical help.

\section{References}

1 Damato B: Developments in the management of uveal melanoma. Clin Exp Ophthalmol 32(6): 639-647, 2004. PMID: 15575836. DOI: 10.1111/j.1442-9071.2004.00917.x

2 Mudhar HS, Parsons MA, Sisley K, Rundle P, Singh A and Rennie IG: A critical appraisal of the prognostic and predictive factors for uveal malignant melanoma. Histopathology 45(1): 1-12, 2004. PMID: 15228438. DOI: 10.1111/j.1365-2559.2004.01874.x

3 Barak V, Frenkel S, Kalickman I, Maniotis AJ, Folberg R and Pe'er J: Serum markers to detect metastatic uveal melanoma. Anticancer Res 27(4A): 1897-1900, 2007. PMID: 17649791.

4 Barak V, Kaiserman I, Frenkel S, Hendler K, Kalickman I and Pe'er J: The dynamics of serum tumor markers in predicting metastatic uveal melanoma (part 1). Anticancer Res 31(1): 345349, 2011. PMID: 21273622.

5 Hendler K, Pe'er J, Kaiserman I, Baruch R, Kalickman I, Barak $\mathrm{V}$ and Frenkel S: Trends in liver function tests: a comparison with serum tumor markers in metastatic uveal melanoma (part 2). Anticancer Res 31(1): 351-357, 2011. PMID: 21273623.

6 Frenkel S, Zloto O, Pe'er J and Barak V: Insulin-like growth factor- 1 as a predictive biomarker for metastatic uveal melanoma in humans. Invest Ophthalmol Vis Sci 54(1): 490-493, 2013. PMID: 23197685. DOI: 10.1167/iovs.12-10228

7 Rubin LA and Nelson DL: The soluble interleukin-2 receptor: biology, function, and clinical application. Ann Intern Med 113(8): 619-627, 1990. PMID: 2205142. DOI: 10.7326/00034819-113-8-619
8 Barak V, Ginzburg M, Kalickman I and Polliack A: Serum soluble interleukin-2 receptor levels are associated with clinical disease status and histopathological grade in non-Hodgkin's lymphoma and chronic lymphocytic leukemia. Leuk Lymphoma 7(5-6): 431-438, 1992. PMID: 1493444. DOI: 10.3109/10428199209049799

9 Marri PR, Hodge LS, Maurer MJ, Ziesmer SC, Slager SL, Habermann TM, Link BK, Cerhan JR, Novak AJ and Ansell SM: Prognostic significance of pretreatment serum cytokines in classical Hodgkin lymphoma. Clin Cancer Res 19(24): 6812-6819, 2013. PMID: 24141626. DOI: 10.1158/1078-0432.CCR-13-1879

10 Barak V, Nisman B, Dann EJ, Kalickman I, Ruchlemer R, Bennett MA and Polliack A: Serum interleukin 1 beta levels as a marker in hairy cell leukemia: correlation with disease status and sIL-2R levels. Leuk Lymphoma 14(Suppl 1): 33-39, 1994. PMID: 7820051.

11 Yoshida N, Oda M, Kuroda Y, Katayama Y, Okikawa Y, Masunari T, Fujiwara M, Nishisaka T, Sasaki N, Sadahira Y, Mihara K, Asaoku H, Matsui H, Seto M, Kimura A, Arihiro K and Sakai A: Clinical significance of sIL-2R levels in B-cell lymphomas. PLoS One 8(11): e78730, 2013. PMID: 24236041. DOI: $10.1371 /$ journal.pone.0078730

12 Goto N, Tsurumi H, Goto H, Shimomura YI, Kasahara S, Hara T, Yasuda I, Shimizu M, Murakami N, Yoshikawa T, Fukuno K, Takahashi T, Kito Y, Takami T and Moriwaki H: Serum soluble interleukin-2 receptor (sIL-2R) level is associated with the outcome of patients with diffuse large B cell lymphoma treated with R-CHOP regimens. Ann Hematol 91(5): 705-714, 2012. PMID: 22183251. DOI: 10.1007/s00277-011-1363-4

13 Kitagawa J, Hara T, Tsurumi H, Goto N, Kanemura N, Yoshikawa T, Kasahara S, Yamada T, Sawada M, Takahashi T, Shimizu M, Takami $\mathrm{T}$ and Moriwaki H: Serum-soluble interleukin-2 receptor (sIL-2R) is an extremely strong prognostic factor for patients with peripheral T-cell lymphoma, unspecified (PTCL-U). J Cancer Res Clin Oncol 135(1): 53-59, 2009. PMID: 18592269. DOI: 10.1007/s00432-008-0440-0

14 Sakai A and Yoshida N: The role of tumor-associated macrophages on serum soluble IL-2R levels in B-cell lymphomas. J Clin Exp Hematop 54(1): 49-57, 2014. PMID: 24942946. DOI: 10.3960/jslrt.54.49

15 Barak V, Schwartz A, Kalickman I, Nisman B, Gurman G and Shoenfeld Y: Hypophosphatemia as a diagnostic tool in sepsis: the role of cytokines. Am J Med 104: 40-47, 1998.

16 Deutsch E, Kaufman M, Nisman B and Barak V: Cytokine evaluation in throat infections. Ann Otol Rhinol Laryngol 107(8): 713-716, 1998. PMID: 9716876. DOI: 10.1177/0003489498 10700815

17 Evereklioglu C, Er H, Türköz Y and Cekmen M: Serum levels of TNF-alpha, sIL-2R, IL-6, and IL-8 are increased and associated with elevated lipid peroxidation in patients with Behçet's disease. Mediators Inflamm 11(2): 87-93, 2002. PMID: 12061429. DOI: $10.1080 / 09629350220131935$

18 Barak V, Selmi C, Schlesinger M, Blank M, Agmon-Levin N, Kalickman I, Gershwin ME and Shoenfeld Y: Serum inflammatory cytokines, complement components, and soluble interleukin 2 receptor in primary biliary cirrhosis. J Autoimmun 33(3-4): 178182, 2009. PMID: 19846277. DOI: 10.1016/j.jaut.2009.09.010

19 Ottaiano A, Leonardi E, Simeone E, Ascierto PA, Scala S, Calemma R, Bryce J, Caracò C, Satriano RA, Gianfranco N, Franco R, Botti G and Castello G: Soluble interleukin-2 receptor in stage I-III melanoma. Cytokine 33(3): 150-155, 2006. PMID: 16517174. DOI: $10.1016 /$ j.cyto.2006.01.002 
20 Saito H, Tsujitani S, Ikeguchi M, Maeta M and Kaibara N: Serum level of a soluble receptor for interleukin-2 as a prognostic factor in patients with gastric cancer. Oncology 56(3): 253-258, 1999. PMID: 10202282. DOI: 10.1159/000011973

21 Gross M, Meirovich A, Rachmut J, Kalichman I, Peretz T, Eliashar R and Barak V: The diagnostic and prognostic value of sIL-2R as an immune biomarker in head and neck cancers. Anticancer Res 36(8): 4347-4352, 2016. PMID: 27466555.

22 Tartour E, Mosseri V, Jouffroy T, Deneux L, Jaulerry C, Brunin F, Fridman WH and Rodriguez J: Serum soluble interleukin-2 receptor concentrations as an independent prognostic marker in head and neck cancer. Lancet 357(9264): 1263-1264, 2001. PMID: 11418153. DOI: 10.1016/s0140-6736(00)04420-2

23 Gilad R, Lampl Y, Eshel Y, Barak V and Sarova-Pinhas I: Cerebrospinal fluid soluble interleukin-2 receptor in cerebral lupus. Br J Rheumatol 36(2): 190-193, 1997. PMID: 9133927. DOI: $10.1093 /$ rheumatology/36.2.190

24 Liu Y, Ho RC and Mak A: Interleukin (IL)-6, tumour necrosis factor alpha (TNF- $\alpha$ ) and soluble interleukin-2 receptors (sIL$2 \mathrm{R})$ are elevated in patients with major depressive disorder: a meta-analysis and meta-regression. J Affect Disord 139(3): 230239, 2012. PMID: 21872339. DOI: 10.1016/j.jad.2011.08.003

25 Al-Hakeim HK, Al-Rammahi DA and Al-Dujaili AH: IL-6, IL18 , sIL-2R, and TNF $\alpha$ proinflammatory markers in depression and schizophrenia patients who are free of overt inflammation. J Affect Disord 182: 106-114, 2015. PMID: 25985379. DOI: 10.1016/j.jad.2015.04.044
26 Levine J, Barak Y, Chengappa KN, Rapoport A, Rebey M and Barak V: Cerebrospinal cytokine levels in patients with acute depression. Neuropsychobiology 40(4): 171-176, 1999. PMID: 10559698. DOI: $10.1159 / 000026615$

27 Levine J, Barak Y, Chengappa KR, Rapoport A, Antelman SM and Barak V: Low CSF soluble interleukin 2 receptor levels in acute depression. Short communication. J Neural Transm (Vienna) 106(9-10): 1011-1015, 1999. PMID: 10599881. DOI: $10.1007 / \mathrm{s} 007020050219$

28 Barak V, Pe'er J, Kalickman I and Frenkel S: sIL-2R an immuno-biomarker for prediction of metastases in uveal melanoma. ARVO Annual Meeting Abstract, IOVS 55, 13, 2014.

29 Frenkel S, Nir I, Hendler K, Lotem M, Eid A, Jurim O and Pe'er $\mathrm{J}$ : Long-term survival of uveal melanoma patients after surgery for liver metastases. Br J Ophthalmol 93(8): 1042-1046, 2009. PMID: 19429579. DOI: 10.1136/bjo.2008.153684

30 Barak V, Pe'er J, Kalickman I and Frenkel S: VEGF as a biomarker for metastatic uveal melanoma in humans. Curr Eye Res 36(4): 386-390, 2011. PMID: 21284506. DOI: 10.3109/ 02713683.2010 .534573
Received January 2, 2022

Revised January 23, 2022

Accepted February 2, 2022 